\title{
Sudden death in a young male with a recent pneumothorax: a case report
}

\author{
To the Editor:
}

An 18-year-old Caucasian male presented to his local doctor with acute left shoulder and interscapular pain. A chest radiograph demonstrated a complex left-sided pneumothorax, which was subsequently confirmed on a thoracic computed tomography (CT) scan (fig. 1). There were multiple septations traversing the pleural space. The patient smoked cigarettes. He denied illicit drug use, including marijuana. His nonconsanguineous parents and one younger male sibling were alive and free from significant medical disease. He had a history of depression for which he was prescribed fluoxetine.

5 months previously he had been admitted to hospital for 10 days with a spontaneous perforated sigmoid colon complicated by faecal peritonitis. The aetiology of the perforation had been unclear, but a CT scan of the abdomen and pelvis, including views of the lung bases, had demonstrated a right-sided pleural effusion. A Hartmann's procedure was performed and was successfully reversed 2 months later. Histology of the resected bowel was unremarkable.

On examination the patient had a tall and slender build, but this was not remarkable. Body mass index and arm span were not formally measured. There was no joint hypermobility, chest wall deformity or corneal abnormality. Palatal examination was normal.

The presumptive diagnosis was of spontaneous left-sided pneumothorax with previous fibrinous pleural disease, which was thought to most likely have arisen as a consequence of an undiagnosed empyema from intra-abdominal sepsis or nosocomial pneumonia 5 months previously. He was treated with i.v. benzylpenicillin and doxycycline, and a small-bore pigtail intercostal catheter was inserted under CT guidance. Subsequently, after removal of the catheter, there was improved, although persisting, left-sided pneumothorax. The patient was discharged after a period of observation. 5 days later at a scheduled outpatient review there was clinical and radiological stability.

3 days later whilst undertaking strenuous activity he developed sudden onset chest pain and shortness of breath. The paramedics were contacted, and on arrival the patient was found to be in shock. There was rapid clinical decline and, despite aggressive attempts at resuscitation, the patient developed pulseless electrical activity en route to hospital. The patient was pronounced dead on arrival at hospital and referred for autopsy.

A post mortem examination revealed a large left haemothorax, haemoperitoneum and retroperitoneal haematoma. Both the aorta and the superior vena cava were thin walled and noted to tear easily with minimal manipulation. Whilst no overt vascular defect was identified, it was concluded that death was due to massive spontaneous bleeding, and a possible diagnosis of vascular or type IV Ehlers-Danlos syndrome (EDS) was questioned.

DNA was extracted from peripheral blood and COL3A1 gene sequencing was performed. A glycine substitution mutation (c.737G >A, pGly246Glu) was identified in the triple helical domain of the gene, confirming the diagnosis of type IV EDS. The patient's parents were reviewed. Both were middle aged, had no significant medical history and on examination demonstrated no joint hypermobility, skin hyperelasticity or other features of type IV EDS. Genetic testing was performed on the patients parents and older sibling none of whom were shown the carry the COL3A1 gene mutation. It was assumed the patient's mutation was de novo and no further familial screening was undertaken.

EDS is characterised by hyperelasticity of the skin and hypermobile joints, and is caused by genetic mutations that result in defects in collagen synthesis or function. It is subdivided into several types according to phenotypic, biochemical and genetic differences. Vascular type (type IV) EDS is a lifethreatening subtype accounting for $\sim 5 \%$ of cases. It results from mutations in the COL $3 A 1$ gene [1], that in turn lead to a reduction in type III pro-collagen structure or function, the precursor to a collagen found in abundance in the aorta and intestines [2]. Mutations are usually acquired either in a heritable fashion via autosomal dominant transmission, or in $\sim 50 \%$ of cases through spontaneous mutation $[3,4]$. Vascular EDS is recognised clinically by the presence of two or more of the following: rupture of blood vessels or internal organs; facial acrogeria; thin translucent skin with highly visible blood vessels and extensive 


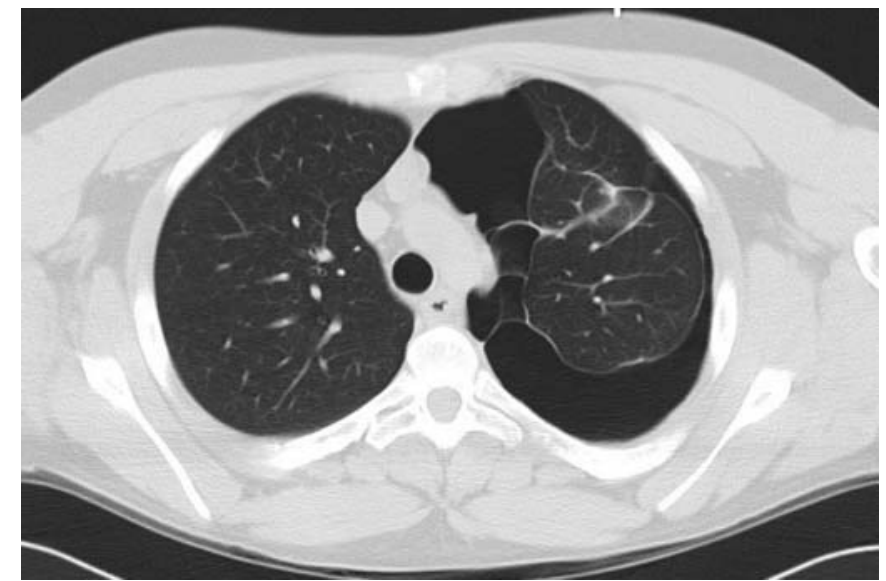

FIGURE 1 Thoracic computed tomography scan showing a complex left-sided pneumothorax.

bruising [5]. Joint changes are less prominent than in other subtypes of the syndrome. The diagnosis can be confirmed either by culture of skin fibroblasts demonstrating a reduced production of type 3 collagen, or by identifying an offending mutation on genetic sequencing. These patients are susceptible to sudden death due to rupture of vascular structures and hollow organs. Pneumothorax is a generally rare manifestation of the syndrome [6], although in one small Japanese series was relatively more common [7]. Other recognised, albeit rare, respiratory complications of vascular EDS include alveolar haemorrhage with haemoptysis and spontaneous haemothorax $[8,9]$.

This case is noteworthy for the subtlety of the diagnostic clinical features. Although in retrospect the patient's parents described a tendency towards easy bruising and increased flexibility of the thumbs, however, facial acrogeria, translucent skin and bruising were not apparent when the patient presented to hospital. Gross joint hypermobility was also absent. No macroscopic or microscopic abnormalities of bowel tissue were noted at the time of previous intra-abdominal surgery or during subsequent histological analysis. The patient underwent both the initial colectomy and subsequent colostomy reversal without complication.

Therefore, this case serves as a salient reminder for clinicians to consider type-IV EDS in patients presenting with otherwise unexplained spontaneous visceral or vascular rupture. It should be considered even in the absence of a relevant family history or marked clinical signs, and also with unusual clinical manifestations such as pneumothorax. In general a diagnosis of vascular type EDS may provide an opportunity for surveillance and surgical planning for index patients and genetic counselling for family members, particularly in the non-spontaneous, autosomal dominant form of the condition. Finally, this case demonstrates the importance of post mortem examination whereby astute autopsy examination can provide invaluable insights into unexpected patient death.

\section{@ERSpublications}

Type IV Ehlers-Danlos syndrome should be considered as a possible diagnosis in patients with spontaneous pneumothorax http://ow.ly/q8u4j

XPATH ERROR: count() function - wrong number of parameters; exactly 1 expected.

${ }^{1}$ Dept of Respiratory and Sleep Medicine, Monash Medical Centre, Melbourne, ${ }^{2}$ Tasmania Dept of Human and Health Services, Royal Hobart Hospital, Hobart, ${ }^{3}$ Tasmanian Clinical Genetics Service, Royal Hobart Hospital, Hobart, ${ }^{4}$ Dept of Medical Imaging, Royal Hobart Hospital, Hobart, ${ }^{5}$ Tasmanian School of Medicine, University of Tasmania, Hobart, and ${ }^{6}$ School of Medicine and Dentistry, Faculty of Medicine, Health and Molecular Sciences, James Cook University, Cairns Base Hospital, Cairns, Australia.

Correspondence: J.B. Geake, Dept of Thoracic Medicine, The Prince Charles Hospital, Rode Road, Chermside, Brisbane 4032, Australia. E-mail: james.geake@health.qld.gov.au

Received: June 282013 | Accepted after revision: Aug 032013

Conflict of interest: Disclosures can be found alongside the online version of this article at err.ersjournals.com

Provenance: Submitted article, peer reviewed.

\section{References}

1 Barabas AP. Vascular complications in the Ehlers-Danlos syndrome, with special reference to the "arterial type" or Sack's syndrome. J Cardiovasc Surg (Torino) 1972; 13: 160-167. 
2 Prockop DJ, Czarny-Ratajczak M. Heritable disorders of connective tissue. In: Fauci AS, Braunwald E, Kasper DL, et al., eds. Harrison's Principles of Internal Medicine. 17th Edn. McGraw-Hill, New York, 2008; pp. 2465-2468.

3 Pepin M, Schwarze U, Superti-Furga A, et al. Clinical and genetic features of Ehlers-Danlos syndrome type IV, the vascular type. N Engl J Med 2000; 342: 673-680.

4 Oderich GS, Panneton JM, Bower TC, et al. The spectrum, management and clinical outcome of Ehlers-Danlos syndrome type IV: a 30-year experience. J Vasc Surg 2005; 42: 98-106.

5 Beighton P, De Paepe A, Steinmann B, et al. Ehlers-Danlos syndromes: revised nosology, Villefranche, 1997. EhlersDanlos National Foundation (USA) and Ehlers-Danlos Support Group (UK). Am J Med Genet 1998; 77: 31-37. Noppen M. Spontaneous pneumothorax: epidemiology, pathophysiology and cause. Eur Respir Rev 2010; 19: 217-219. Watanabe A, Kosho T, Wada T, et al. Genetic aspects of the vascular type of Ehlers-Danlos syndrome (vEDS, EDSIV) in Japan. Circ J 2007; 71: 261-265.

8 Ayres JG, Pope FM, Reidy JF, et al. Abnormalities of the lungs and thoracic cage in the Ehlers-Danlos Syndrome. Thorax 1985; 40: 300-305.

9 Purohit N, Marsland D, Roberts N, et al. Haemo-pneumothorax and haemoptysis in a patient with suspected Ehlers-Danlos Syndrome. Interact Cardiovasc Thorac Surg 2009; 9: 130-131.

Eur Respir Rev 2014; 23: 145-147 | DOI: 10.1183/09059180.00004913 | Copyright ()ERS 2014 ERR articles are open access and distributed under the terms of the Creative Commons Attribution Non-Commercial Licence 3.0. 